\title{
JUURNAL.RU
}

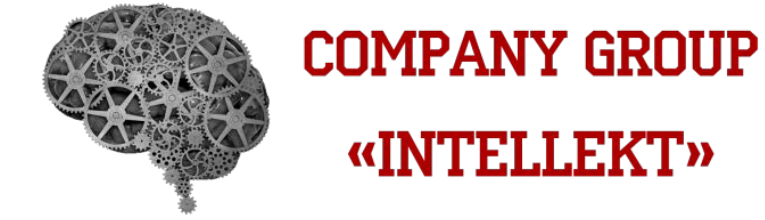

Нсуе ХасинтоМба Бийе, Федосов В.П. Южный федеральный университет Таганрог, Россия

doi: 10.18411/lj2016-6-3-05

\section{Цифровой алгоритм измерения кратковременной нестабильности частоты высокостабильных генераторов умножительно-преобразовательным методом}

Введение. Кратковременная нестабильность высокостабильных генераторов кварцевых генераторов, обеспечивающих синхронизацию цифровых сетей связи, составляет 10-13 (за 2 с). Оценка такой нестабильности не простая задача, поскольку измерительные приборы, которые измеряют частоту, имеют, как правило, погрешность 10-8 - 10-9 (за 2 с) и не позволяют оценить возможные отклонения частоты таких генераторов. Для решения такой задачи может быть применен умножительно-преобразовательный метод, реализованный в цифровом виде.

Структура метода представлена на рисунке 1. Исследуемые независимые генераторы Г1 и Г2 формируют гармонические колебания одинаковой частоты и нестабильности, в которых обеспечивается исключение явления захвата частоты; колебания преобразуются в цифровые отсчеты, которые возводятся в 4ю степень (на выходе Г1) и в 5-ю степень (на выходе Г2); полученные колебания фильтруются полосовыми фильтрами, настроенными на 4 гармонику исходного колебания в полосовом фильтре ПФ1 и на 5-ю гармонику в полосовом фильтре ПФ2; колебания на выходах полосовых фильтров складываются и сумма возводится в 2-ю степень. 


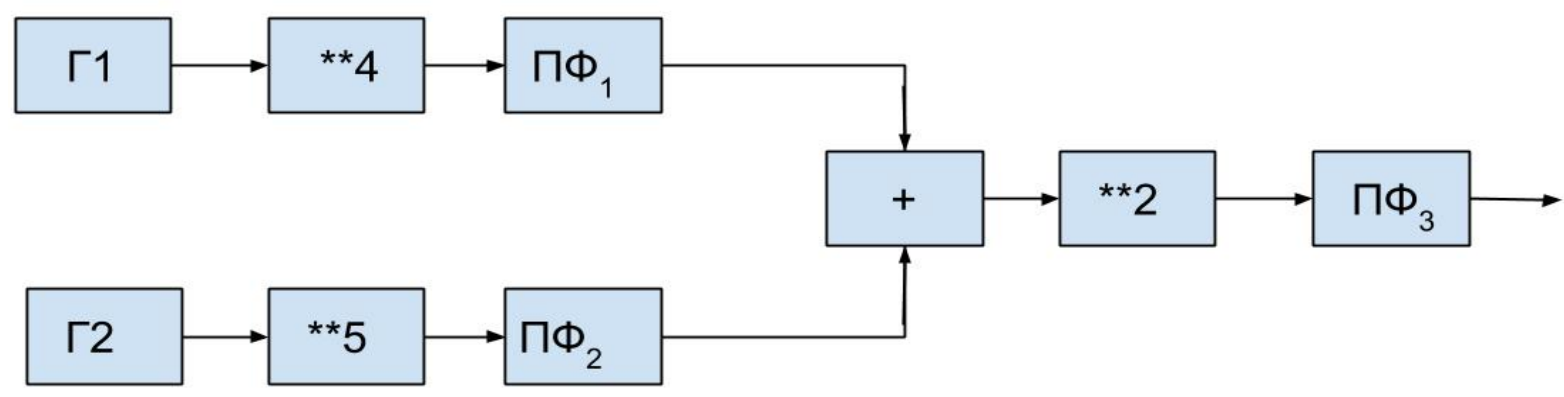

Рисунок 1 - Структура последовательности умножительно-преобразовательных операций для кратковременной оценки нестабильности частоты идентичных по частоте и нестабильности генераторов

Этот сигнал затем фильтруется в полосовом фильтре ПФ3, настроенном на комбинационную составляющую, по частоте равную частоте исследуемых генераторов. При таких преобразованиях нестабильность частоты возрастает в 41 раз по дисперсии или в $\sqrt{41}$ раз по среднеквадратическому отклонению. Применяя такую умножительно-преобразовательную операцию несколько раз, можно увеличить результирующую нестабильность частоты до такой величины, которую можно оценить обычными измерителями частоты, а затем пересчитать эту нестабильность для использованных генераторов, учитывая их идентичность.

Разработанная программа на языке графического программирования LabVIEW, реализующая представленный метод в виде алгоритма, показана блок-диаграммой на рисунке 2, а ее лицевая панель представлена на рисунке 3.

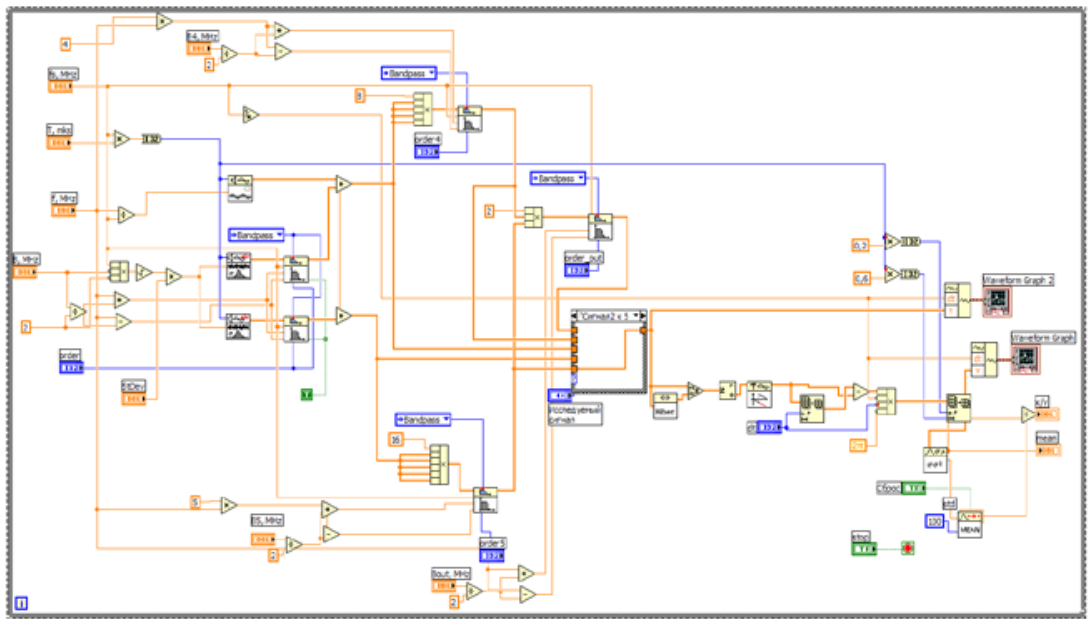

Рисунок 2 - Программа на LabVIEW умножительно-преобразовательной ячейки алгоритма для оценки нестабильности по частоте двух идентичных высокостабильных генераторов 
На лицевой панели представлено выходное гармоническое колебание, основная гармоника внизу, а сверху - узкополосный шум.

Элементы управления представлены вертикально сверху вниз на лицевой панели: частота дискретизации сигнала при моделировании равна 100 МГц; длительность реализации колебания 10 мс при моделировании, частота колебания - 1 МГц, в результате получается 100 отсчетов на один период колебания, то есть с большим запасом выполняется теорема Котельникова. Полоса пропускания полосового фильтра, который формирует узкополосный шум, равна 0,01 МГц; порядок формирующего шум фильтра (order) установлен равным 2; стандартное СКО шума на выходе этого полосового фильтра равно 0,001 В; интервал дискретизации 5 мкс; полоса первого полосового фильтра ПФ1 (см. рис. 1) составляет 0,1 МГц и порядок равен 4 (B4 и Order 4); соответственно для второго полосового фильтра (B4, Order 5)полоса и порядок равны 0,1 МГц и 5; а для фильтра ПФ3 (В_out и Order_out) полоса равна 0,2 МГц и порядок - 6. Далее имеется переключатель "исследуемый сигнал" (см. рис. 3) для отображения 5 сигналов, она имеет 5 входов и один выход: сигнал 1, сигнал 2 , сигнал, возведенный в 4 степень, сигнал, возведенный в 5 степень и выходной сигнал; поэтому сигнал на выходе этой структуры зависит от выбора. Последняя функция - измеритель, который включает в себя три индикатора, которые показывают следующие параметры: среднеквадратическое отклонение частоты "standard deviation", среднее значение частоты "mean" и отношение среднеквадратического отклонения частоты к среднему ее значению "х/y".

Необходимо обратить внимание на то, что на лицевой панели рисунка 3 представлены только выходной сигнал и шум, который к нему добавляется.

Заключение. Таким образом, в статье представлен алгоритм, позволяющий на основе умножительно-преобразовательного метода производить оценку кратковременной нестабильности частоты 
высокостабильных генераторов. Результаты преобразований показывают, что среднеквадратическое отклонение частоты возрастает в $\sqrt{41}$ раз.

Авторы выражают благодарность Цветкову Федору Алексеевичу за помощь в разработке программы на LabVIEW.

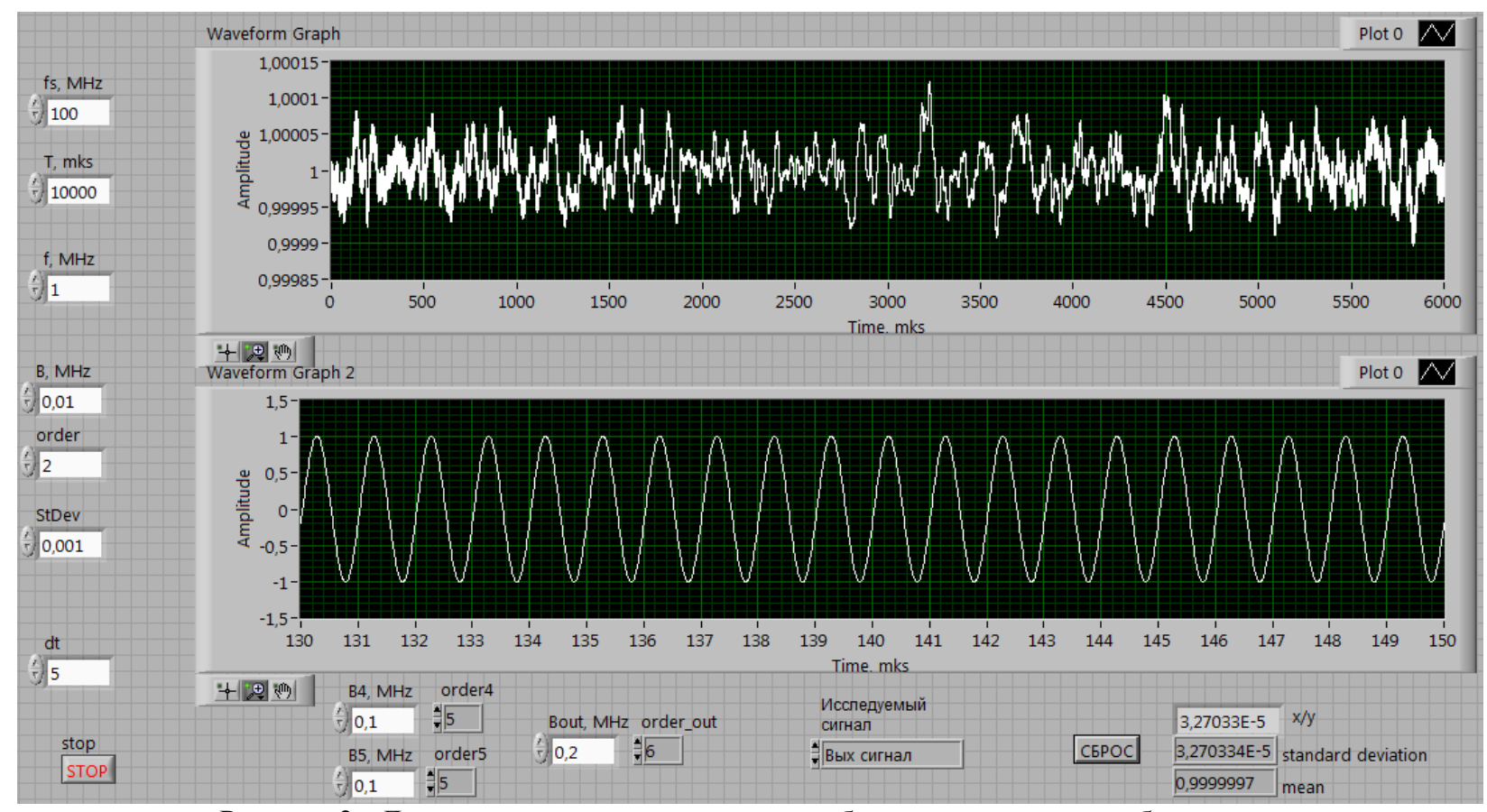

Рисунок 3 - Лицевая панель виртуального прибора для оценки нестабильности

высокостабильных по частоте идентичных генераторов умножительно-преобразовательным способом 